\title{
Some Experimental Aspects of Absolute Configuration Determination using Single Crystal X-ray Diffraction ${ }^{\text {ts }}$
}

\author{
Amber L. Thompson ${ }^{\mathrm{a}, *}$, Sarah F. Jenkinson ${ }^{\mathrm{b}}$, George W. J. Fleet ${ }^{\mathrm{b}}$ \\ ${ }^{a}$ Chemical Crystallography, Chemistry Research Laboratory, \\ Mansfield Road, Oxford. OX1 3TA \\ ${ }^{b}$ Chemistry Research Laboratory, Mansfield Road, \\ Oxford. OX1 3TA
}

\begin{abstract}
Students of single crystal X-ray diffraction are often give advice as to how best to collect their data when attempting absolute configuration determination. These "rules" often have more grounding in gut-feeling than evidence. Thus, in an effort to provide advice and evidence that today's crystallographers can pass onto to tomorrow's young scientists, we present a systematic study of 1-deoxy-L-arabinitol, a straight chain sugar which crystalises well in the space group $\mathrm{I}_{1}$.
\end{abstract}

Keywords: Absolute configuration determination, Absolute structure determination, Flack " $x$ " parameter, 1-deoxy-L-arabinitol, tartaric acid,

\section{Introduction}

Optical activity, the direction of rotation of the plane of polarised light is the oldest known manifestation of molecular chirality and still the most widely used and understood. A wide range of other techniques are now also available including circular dichroism, chiral chromatography, and chiral separation methods, but single crystal diffraction is perhaps the most powerful solid-state technique. For the organic chemist, the problem of interest is the determination of the absolute configuration, i.e. the spatial arrangement of

\footnotetext{
${ }^{2}$ Contributed in Memory of Howard Flack.

*Corresponding author: amber.thompson@chem.ox.ac.uk
} 
the atoms of a chiral molecule and its stereochemical description. This is closely tied up with the crystallographic problem of absolute structure, the solution of which gives rise to the absolute configuration in the solid state.

Essentially, a mixture of enantiomers can crystalise in two ways: as a conglomerate, where molecules have spontaneously resolved and each crystal is enantiopure; or as a racemate, where opposite hands of the substrate are related by crystal symmetry. The first of these, the conglomerate, can only in crystalise a non-centrosymmetric space group which additionally contains no mirror symmetry or improper rotation. Sixty-five of the total two-hundred and thirty possible space groups are these so-called Sohnke Space Groups. ${ }^{1}$ Chiral materials crystallising in the non-Sohnke Space Groups give rise to the second category, the racemates, where crystallographic inversion, mirror or improper rotation generate the second enantiomer ensuring that the bulk crystal is rigourously racemic. In this way, the thirty-two possible geometric crystal classes can be divided to reflect the chirality (Fig. 1).

There are two additional cases. The first of these is the case where the conglomerate crystalises as a twin, and the crystal comprises domains that have spontaneously resolved and are of different enantiomers. In this case, the enantiomeric composition will depend on the twin ratio, i.e. the ratio of the volume of the two different components. The final case is the unusual situation where a chiral compound has crystalised in a Sohnke Space Group (i.e. without inversion, mirror or improper rotation symmetry), but has two molecules in the asymmetric unit $\left(Z^{\prime}=2\right)$, one of each enantiomer. These are sometimes referred to as "chiral racemates" or "kryptoracemates" and there are less than two hundred known. ${ }^{2}$ In this case, the crystal is by definition racemic, though it may also be twinned by inversion. Higher orders of $Z^{\prime}$ are also possible in principle, but in general only even numbers will be racemic crystals. $^{\dagger}$ Howard Flack was interested in many aspects of chemistry and crystallography, but he was particularly concerned with chirality and the problem of absolute structure: statistically distinguishing the enantiopure single crystal from the case of the inversion twin.

In a centrosymmetric crystal, reflections or diffraction "spots" related by inversion symmetry should have the same intensity. This is known as

${ }^{\dagger}$ For $Z^{\prime}=2 n$, since the first twin domain is racemic, then the second will also be racemic and $\mathrm{D}$ will become $\mathrm{L}$ and vice versa. Where $Z^{\prime}$ is odd, the percentage of each enantiomer present will depend on the value of $Z^{\prime}$ and the twin ratio. 


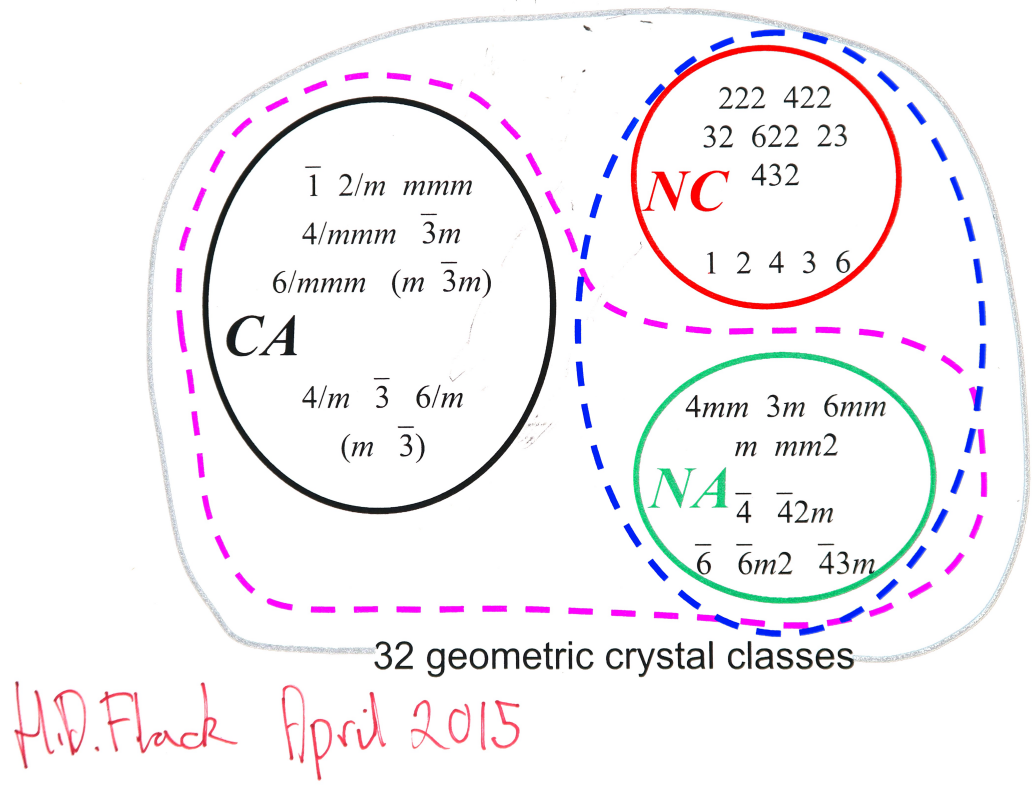

Figure 1: A slide taken from a presentation given by Howard Flack in Oxford in 2005, which was stuck on the wall in the laboratory, and signed by him 10 years later on another visit to Chem. Cryst. in 2015. The non-centrosymmetric classes are in the group marked with a broken blue line and the non-Sohnke clases are marked with a broken pink line. The non-intersecting subgroups of these can be denoted "NC" (non-centrosymmetric chiral; i.e. Sohnke space groups) and "CA" (i.e. centrosymmetric achiral). The intersection can be labeled "NA", the non-centrosymmetric achiral classes. As Flack went on to comment in the presentation it is the "NA" set in particular that often give rise to confusion and mistakes. The image was originally printed in black and white has been re-coloured to match the slide given in his presentation. 
Friedel's Law (Eqn. 1). ${ }^{3}$ In an enantiopure single crystal reflections do not obey Friedel's Law, though the differences between the intensities of the Friedel pairs will be tiny. In general, the magnitude of the differences will depend on the atomic number of the heaviest atom, which is why it is often said that, "to determine the absolute configuration, you need a bromine". The magnitude of the Friedel differences also depend on the wavelength, $\lambda$, of the incident radiation used, however. For longer wavelengths and heavier atoms the Friedel difference increases, but it typically remains of the order of the expected standard uncertainty of the observation.

$$
I_{h k l} \approx I_{\bar{h} \bar{k} \bar{l}}
$$

In principle, however, if for a given chiral compound, the intensity of reflection $h k l$ is greater than that of its Friedel pair, $\bar{h} \bar{k} \bar{l}, I_{h k l}$ will be less than $I_{\bar{h} \bar{k} \bar{l}}$ for its enantiomer. This simple theory was first used for absolute structure determination over sixty-five years ago by Bijvoet, Peerdeman and van Bommel. ${ }^{4}$ Bijvoet and co-workers carried out careful measurements of just fifteen pairs of reflections from on sodium rubidium tartrate using a Weissenberg camera and a specially constructed zirconium X-ray tube ( $\lambda=0.786 \AA$; i.e. close to the Rb K-absorption edge at $0.865 \AA$ to enhance the anomalous scattering and increase the differences in intensities). This method, together with the Hamilton $R$-factor ratio test, ${ }^{5}$ was used for thirty years, until Rogers introduced his $\eta$ parameter, ${ }^{6}$ a least-squares parameter, which refined a multiplier onto the imaginary part of the anomalous scattering factor. In turn, this was quickly superseded by the Flack " $x$ " parameter, ${ }^{7}$ which treated the crystal as a mixture of the original enantiomer and its twin by inversion. The Rogers $\eta$ parameter varied between +1 and -1 , and had no physical meaning as it approached the mid point, zero. In contrast, the Flack parameter had a physical meaning over its entire range, with 0 indicating that the structure is the correct hand, a value of 1 indicating it should be inverted and a value near the middle indicating that the sample was twinned by inversion and contained both enantiomers.

The application of Bayesian analysis of the Friedel differences ${ }^{8,9,10}$ allows post-refinement calculation of the absolute structure parameter, known as the Hooft " $y$ " parameter. The analysis described by Hooft et al. also enables the determination of the probability that the presented hand is correct given that the material is known to be enantiopure (the P2 probability) and the probability that the presented hand is correct given that the material is 
known to be either enantiopure or racemic (the P3 probability). Although these can be indicative, and there have been several other attempts to get more information from weak data, ${ }^{11,12}$ the Flack " $x$ " parameter is still the a reliable indicator of the robustness of the absolute structure determination and of the general quality of the analysis and by many considered the "gold standard" $\ddagger$ The actual magnitude of the Flack " $x$ " parameter is therefore clearly important, but it is meaningless without a sufficiently small standard uncertainty, values that were quantified by Flack and Bernardinelli in 2008. ${ }^{14}$ Since then, there has been a propagation of myths suggesting what should be done to get a low standard uncertainty and as a result it is common to hear people say things like, "A redundancy of twenty is necessary to determine the absolute configuration of a light atom structure." It was to assess the reliability of some of these rumours and "rules of thumb" that a series of experiments were carried out.

\section{Results \& Discussion}

\subsection{Tartaric Acid}

Ever since Pasteur's seminal works,${ }^{15,16}$ tartrates have been the archetypal chiral materials. A comparison of refinement results for data collections on a single crystal of D-tartaric acid (space group P2 $2_{1}$; Fig. 2 ) indicate that where the point group used to calculate the data collection strategy is $2 / m$ (i.e. few or no Friedel pairs were measured), the Flack " $x$ " parameter, determined by the original method ${ }^{7}$ refines to $0.1(4)$. Although the standard uncertainty improves considerably when more equivalents of data are collected, the Flack " $x$ " remains at a similar value (Table 1 ). This is unsurprising as it has been shown previously that for samples of known enantiopurity, even a statistically poor determination of the absolute structure tends to give a Flack " $x$ " parameter in-keeping with the expected result. ${ }^{17}$

For each tartaric acid data collection, the Hooft " $y$ " parameter was also calculated and gave a similar result to the Flack " $x$ " parameter, though its standard uncertainty is significantly smaller. This is an observation that

\footnotetext{
†There are now several other methods to determine the absolute structure or Flack " $x$ " parameter, including the so-called "Classical Fit" (formerly known as the "Hole-inOne" ${ }^{13}$ and the Parsons' Quotient Method. ${ }^{12}$ While the conclusions drawn herein may also be applicable to these cases, throughout this study the Flack " $x$ " parameter was determined by full matrix least-squares refinement as described in the original work. ${ }^{7}$
} 


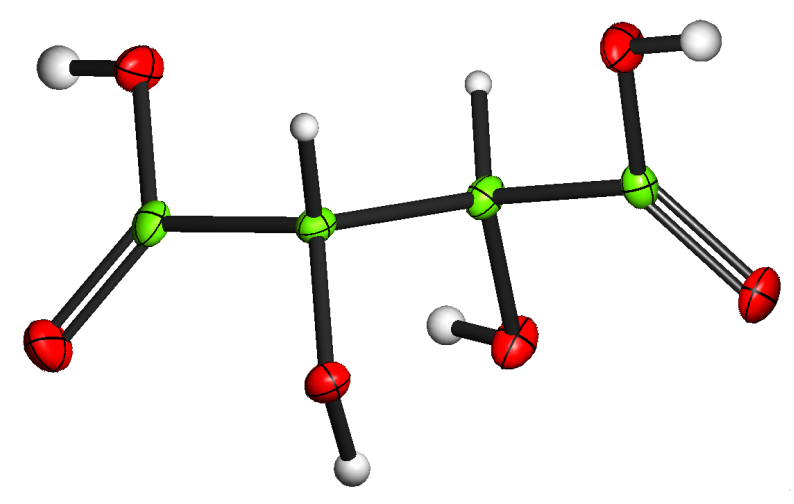

Figure 2: Displacement ellipsoid plot of D-tartaric acid from a full sphere of data collected at $150 \mathrm{~K}$ drawn at $50 \%$ probability.

has been discussed at length recently by Watkin and Cooper. ${ }^{13}$ The P2 and P3 probabilities are strongly indicative of the correct enantiomer as would be expected in the case where the Flack " $x$ " and Hooft " $y$ " parameters are well determined, but they also improve for the more highly redundant data collections.

It is clear, both from these results and from previous reports, ${ }^{18}$ that the Flack " $x$ " parameter is surprisingly robust and that it is the standard uncertainty that is generally affected by collecting additional data. The question of interest here is how much additional data? In a small molecule single crystal laboratory, instrument time is often in particularly high demand. In such facilities, time wasted due to unnecessary collection of "redundant" data is a poor use of a valuable resource. To this end, a systematic study of 1deoxy-L-arabinitol was carried out to follow the behaviour of the standard uncertainty of the Flack " $x$ " parameter.

\subsection{A Variable Temperature Study of 1-deoxy-L-arabinitol}

1-Deoxy-arabinitol (Fig. 3) is a straight chain sugar that crystalises in the space group I4 $4_{1}$ with a pseudo $a$-glide. ${ }^{19}$ The crystals are generally of good quality and this, together with the high crystal symmetry means it is both easy and relatively quick to get high redundancy. Single crystal diffraction data were therefore collected with copper $\mathrm{K}_{\alpha}$ radiation assuming the point group $4 / m, 4,2$ and 1 , at $300 \mathrm{~K}, 250 \mathrm{~K}, 200 \mathrm{~K}, 150 \mathrm{~K}, \& 100 \mathrm{~K}$ and also varying the acquisition time at $150 \mathrm{~K}$ (see Section 4 for more details). The 


\begin{tabular}{lccc}
\hline Point Group & $2 / m$ & 2 & 1 \\
\hline Average Redundancy & 1.4 & 2.1 & 4.5 \\
Data Colleciton Time & $47 \mathrm{mins}$ & $1 \mathrm{hr} 45 \mathrm{mins}$ & $3 \mathrm{hr} 31 \mathrm{mins}$ \\
$<I / \sigma(I)>$ & 38.77 & 51.65 & 68.55 \\
No. of Bijvoet pairs & 182 & 519 & 522 \\
Flack " $x$ " & 0.102 & -0.271 & -0.111 \\
$\sigma(x)$ & 0.417 & 0.262 & 0.183 \\
Hooft " $y$ " & 0.116 & -0.238 & -0.072 \\
$\sigma(y)$ & 0.190 & 0.074 & 0.062 \\
P2(correct) & 1.000 & 1.000 & 1.000 \\
P3(correct) & 0.865 & 1.000 & 1.000 \\
P3(racemic) & 0.135 & 0.000 & 0.000 \\
$R_{1}(I>-3 \sigma(I))$ & $3.87 \%$ & $3.35 \%$ & $2.69 \%$ \\
\hline
\end{tabular}

Table 1: Refinement results for D-tartaric acid where different point groups are used to calculate the data collection strategy. The average redundancy is calculated based on the non-centrosymmetric point group, 2. "P2(correct)" is the probability that the presented hand is correct given that the material is known to be enantiopure; "P3(correct)" is the probability that the presented hand is correct given that the material is known to be either enantiopure or racemic; "P3(racemic)" is the probability that the crystal is racemic given that the material is known to be either enantiopure or racemic. 

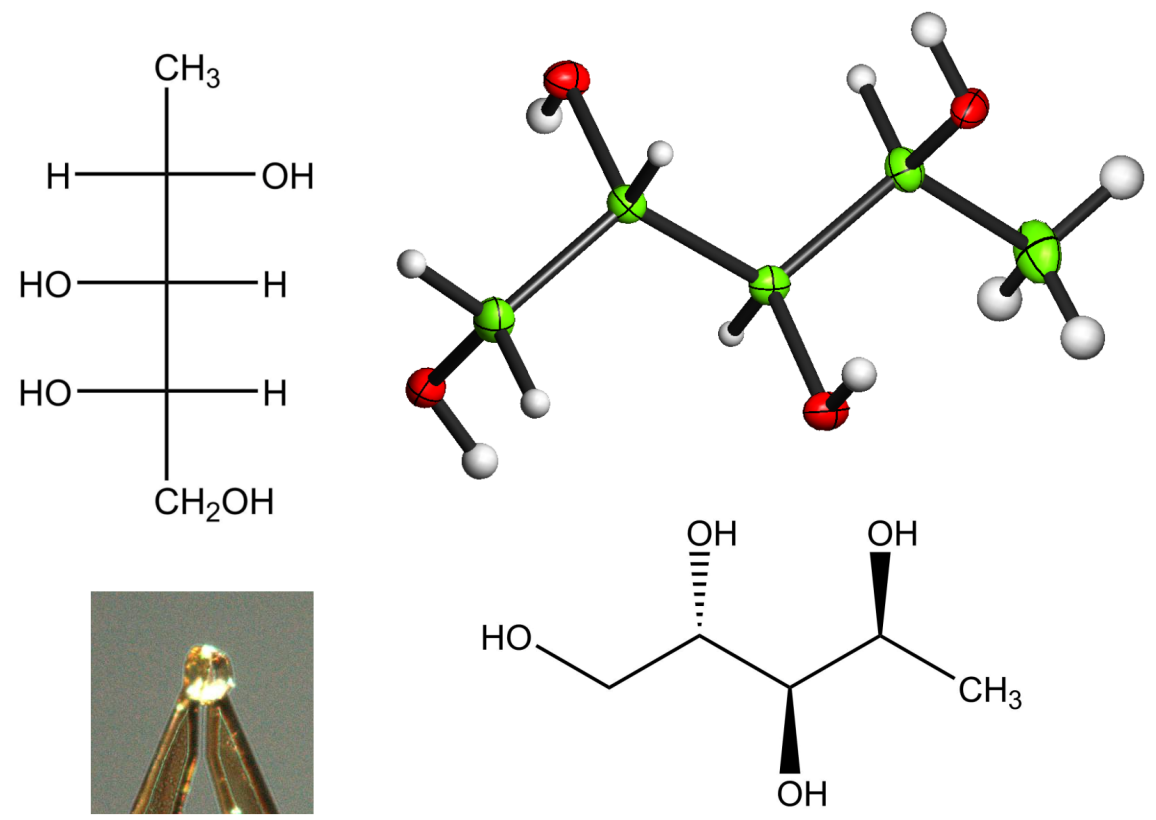

Figure 3: Displacement ellipsoid plot of 1-deoxy-L-arabinitol from a full sphere of data collected at $100 \mathrm{~K}$ drawn at $50 \%$ probability shown together with the Fischer and Natta projections of the stereochemistry and the crystal used for the data collection. 


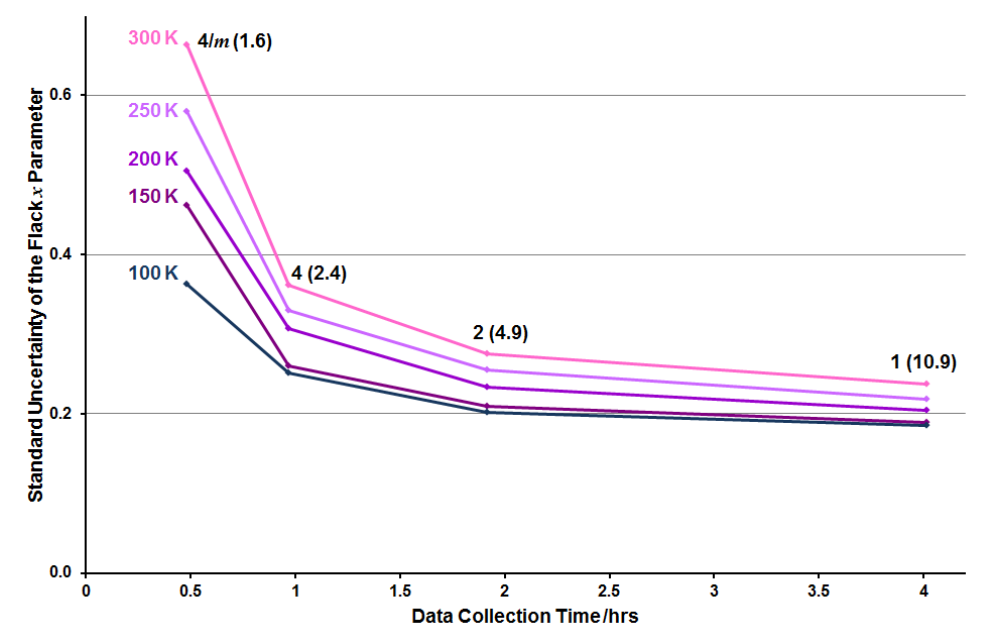

Figure 4: The standard uncertainty of the Flack " $x$ " parameter for datasets with different redundancies and at different temperatures; The point group of the data collection is given with the redundancy based on the point group 4 in parentheses.

data were processed in a highly automated way in order to simulate the nonexpert user and to try to ensure that the data were treated as systematically as possible throughout.

Comparison of the standard uncertainty for each evaluation of " $x$ " showed the variation with temperature as well as the effect of increasing the redundancy (Fig. 4). It was clear that as shown for the tartaric acid above, increasing the redundancy reduced standard uncertainty. It was also clear that making an effort to collect all the Friedel pairs makes a big difference to the standard uncertainty and this accounts for the large difference between the first two data points at each temperature. Thereafter, increasing the amount of data collected has an increasingly diminishing influence on the standard uncertainty as the curve approaches an asymptote at around 0.2.

It was also clear that collecting data at lower temperatures improves the determination of the Flack " $x$ " parameter as the standard uncertainty is reduced. There are a number of factors that could be influential here; for example, decreasing the temperature reduces any tendency to vibrational disorder. The crystal lattice for 1-deoxy-arabinitol comprises strong intermolecular hydrogen bonds, so the disorder is minimal for this material. Reducing the temperature reduces the amount of atomic motion though, reducing the 
Debye-Waller effect and leading to an increase in the intensity of data collected at high angle. ${ }^{20}$ This is apparent from the mean $I / \sigma(I)$ for the high angle data shell $(0.82-0.79 \AA)$ which is 16.51 at $300 \mathrm{~K}$ and 41.36 at $100 \mathrm{~K}$ (the same for the whole dataset collected at $300 \mathrm{~K}$ ). Although the X-ray scattering factors decrease as a function of $\sin \theta / \lambda$, the anomalous scattering terms that give rise to the Friedel differences are independent of $\theta .^{21}$ This means that although the Bijvoet intensity ratio for any material increases at high angle, the Friedel intensity difference does not, ${ }^{22}$ so that increasing the $I / \sigma(I)$ by other means, like reducing the temperature is a potentially useful approach. Thus, barring issues with phase transitions, it is generally a good idea to collect data at the lowest temperature possible as, in general, there is little additional expense involved in running a sample at $100 \mathrm{~K}$ rather than $200 \mathrm{~K}$ for example and the improvement could be significant.

\subsection{Varying the Acquisition time for 1-deoxy-L-arabinitol}

To investigate the effect the mean $I / \sigma(I)$ has on the standard uncertainty of the Flack " $x$ " parameter, the data collected at $150 \mathrm{~K}$ described above were compared with data where the images were collected for twice as long, four times as long and with the generator at half power (to simulate half as long). From these results (Fig. 5) similar features are clearly visible: in each case, the first data point is from data collected assuming $4 / m$ symmetry and in all cases, the curve approaches an asymptote at around 0.2 . It is also apparent that increasing the $\langle I / \sigma(I)\rangle$ improves the standard uncertainty of the Flack " $x$ " parameter at a given redundancy. The magnitude of this improvement depends on the intensity, however, with a much larger improvement at low intensity.

Plotting these data as a function of time (Fig. 6) shows how expensive that improvement is in terms of data collection time. For example there is only a relatively small improvement when a full sphere of data are collected at $4 \mathrm{~s} \& 16 \mathrm{~s}$ compared with the result seen for a hemisphere collected with $2 \mathrm{~s} \& 8 \mathrm{~s}$ per image (which takes less than a third of the time). On the other hand, it is clear that if the data have insufficient intensity, collecting for longer can have a significant impact. Compare for example, the full sphere collected at low power with the hemisphere collected for twice as long and with the generator at full power - the effective time is approximately doubled, but the standard uncertainty shows a marked improvement and it could be argued that the time is well spent. 


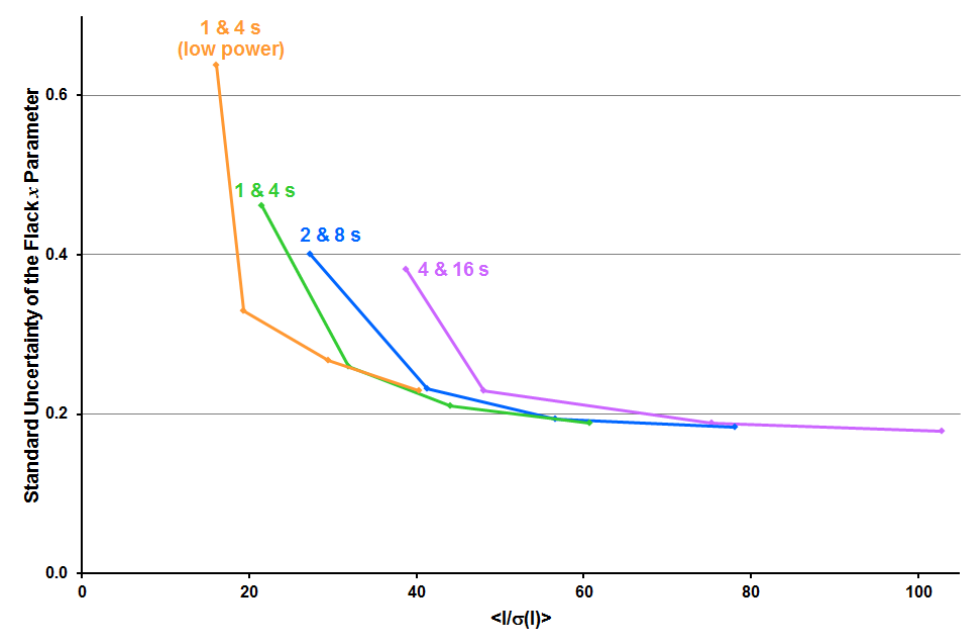

Figure 5: The standard uncertainty of the Flack " $x$ " parameter for datasets with different $\langle I / \sigma(I)\rangle$. The $\langle I / \sigma(I)\rangle$ is the actual value calculated for the whole data collection after data reduction in $\mathrm{I} 4_{1}$ using CrysAlisPro. The acquisition times are give for the low and high angle runs.

In terms of where the time is best spent, it also seems apparent that it is better to spend time collecting "redundant data" rather than increasing the exposure time per image. This is apparent, for example, in the observation that the standard uncertainty for the quarter-sphere collected with $4 \mathrm{~s} \& 16 \mathrm{~s}$ is larger than that for the hemisphere data collected with $2 \mathrm{~s} \& 8 \mathrm{~s}$ (Fig. 6). This only holds true, however, if the intensity is above a certain threshold.

\subsection{The Flack " $x$ " Parameter for 1-deoxy-L-arabinitol}

The results comparing the standard uncertainty of the Flack " $x$ " parameter are very instructive, but this is without considering the value of the parameter itself. In general, it has been observed previously that the Flack " $x$ " parameter is surprisingly robust, and tends to give the correct value even when the standard uncertainty is large. ${ }^{23}$ Indeed that was the result seen above for the tartaric acid example (Section 2.1). In the case of 1-deoxy$\mathrm{L}$-arabinitol, the spread of values for the Flack " $x$ " parameter seen over all the data collected is much larger than is usually to be expected (Fig. 7). On close inspection, however, the worst offenders are all the datasets collected using the strategy calculated assuming $4 / m$ symmetry. 


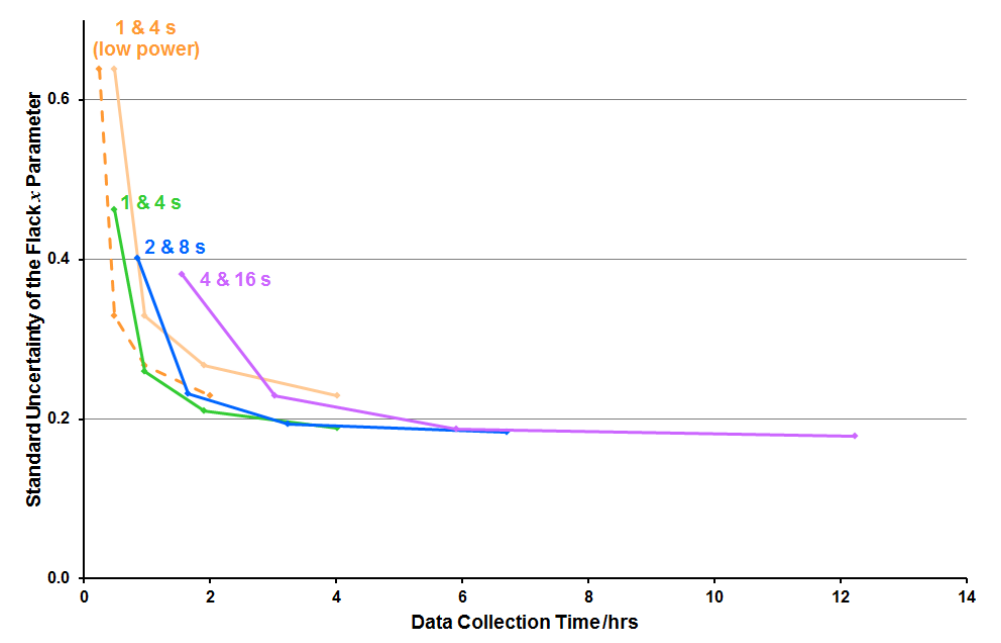

Figure 6: The standard uncertainty of the Flack " $x$ " parameter for datasets collected with different acquisition times. The "low power" data collection appears twice - once with a solid line in its actual position and once with a broken line where the data collection time has been divided by a factor of two.

This is unusual because although having a complete set of Freidel pairs is undoubtedly useful, ${ }^{\S}$ they are not actually required when refining the Flack " $x$ " parameter. ${ }^{18}$ In the case of 1-deoxy-L-arabinitol, it is possible that that problem lies in the pseudo-centrosymmetric nature of the structure. The structure crystallises in the space group $\mathrm{I}_{1}$, but the molecule has a pseudo inversion centre which lies between $\mathrm{C} 2$ and C3 (Fig. 8). This lies at approximately $\frac{3}{4}, \frac{1}{2}, z$ which corresponds well with the inversion centre in $\mathrm{I}_{1} / a$ and gives an $88 \%$ fit. In fact, the structure agrees so well with this higher symmetry that despite repeated efforts, solving the structure with SupERFuIP gives only $\mathrm{I}_{1} / a$ as a possibility, and refinement of this model in this space group has an $R_{1}$ of $6.5 \%$. The structure is identifiable as $\mathrm{I}_{1}$ rather than $\mathrm{I}_{1} / a$, however, because the systematic absences due to the $a$-glide are clearly not absent (Fig. 8). Although these reflections are very obvious for the full sphere data, additional noise reduces their influence for the data collected at higher temperature, with a lower acquisition time, or especially with a low

${ }^{\S}$ Friedel pairs are useful as they can reduce the effect of some systematic errors and the effective signal is twice that for unpaired reflections. 

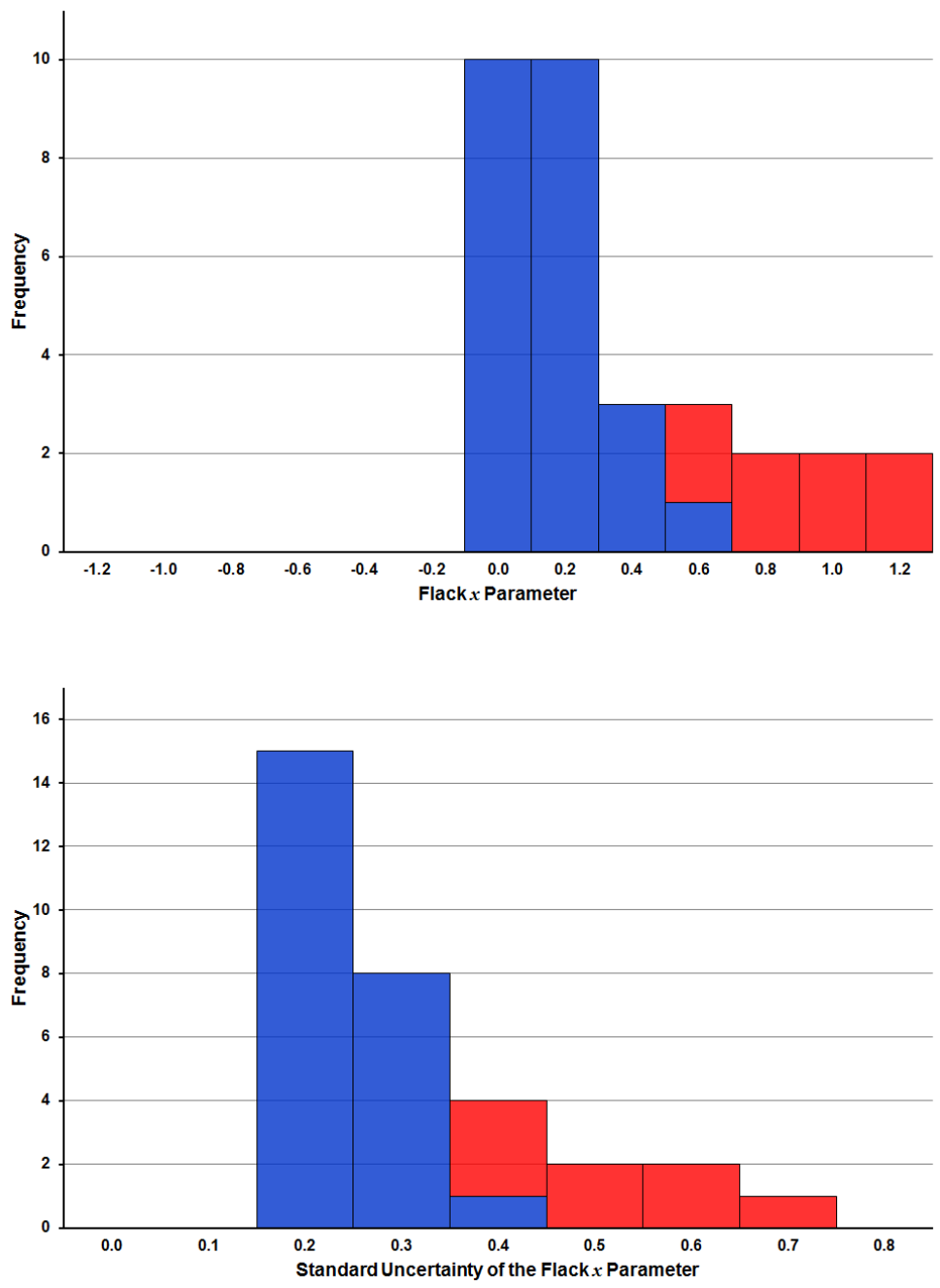

Figure 7: The distributions of the Flack " $x$ " parameter and its standard uncertainty for all the datasets collected on 1-deoxy-L-arabinitol in this study. The datasets collected using the strategy calculated assuming $4 / m$ symmetry are shown in red and are clearly suggesting the structure may be incorrect. 
redundancy. Flack et al. ${ }^{24}$ observed that Friedel coverage is more important where there is pseudo symmetry and in the case of 1-deoxy-arabinitol, this is certainly true as their absence makes the refinements unstable until the weighting scheme is optimised.

There is another possibility, however. I $4_{1}$ is a polar space group, and has a floating origin in $z$. As explained by Cruickshank and MacDonald, ${ }^{25}$ for a non-centrosymmetric structure systematic errors can be introduced along the polar axis when data are missing in that direction. While data were commonly collected this way in the days of serial detectors, this is relatively rare in the modern era of area detectors, but it can happen if the crystal was aligned with one of the axes of the diffractometer (this was not the case here). Examining the $4 / m$ data with a reciprocal lattice view shows that there is a noticeable lack of reflection data in the $c$ and $a$ directions. This is presumably caused by the need to obtain data as efficiently as possible leading to data collected predominantly on one side of reciprocal space.

\section{Conclusions}

It is clear from this study that where instrument time is at a premium, collecting a highly redundant dataset is poor use of resources. For example the full sphere data collection on 1-deoxy-L-arabinitol using $4 \mathrm{~s}$ for the low angle data and $16 \mathrm{~s}$ for the high angle data gives only slightly better results than the data collected in a third of the time. All this said, there is also a word of warning: there is a temptation to focus on the standard uncertainty of the Flack " $x$ " parameter, however, it is clear that under certain conditions such as those described herein, insufficient data can give a misleading result. Clearly then, as in all things, there is a balance to be sought. Bernardinelli and Flack ${ }^{18}$ demonstrated that a complete set of the Friedel pairs are not actually necessary to determine the absolute structure, though Friedel coverage is more important where there is pseudo-symmetry. ${ }^{24}$ From the results herein, which concern one of the most challenging examples of absolute structure determination as it involves a light-atom structure $\left(\right.$ Friedif $\left.^{21}=36.75\right)$, with a pseudo-inversion centre, it is obvious that it is wise to make an concerted effort to obtain as near to a complete set of Friedel pairs as possible to avoid the potential for a misleading result. In a more routine case (like the tartaric acid also discussed), this may not be necessary, however, it is not possible to know whether one has been misled without having a more reliable result to compare it with. Thus, in general, it is wise to err on the side of caution, but 

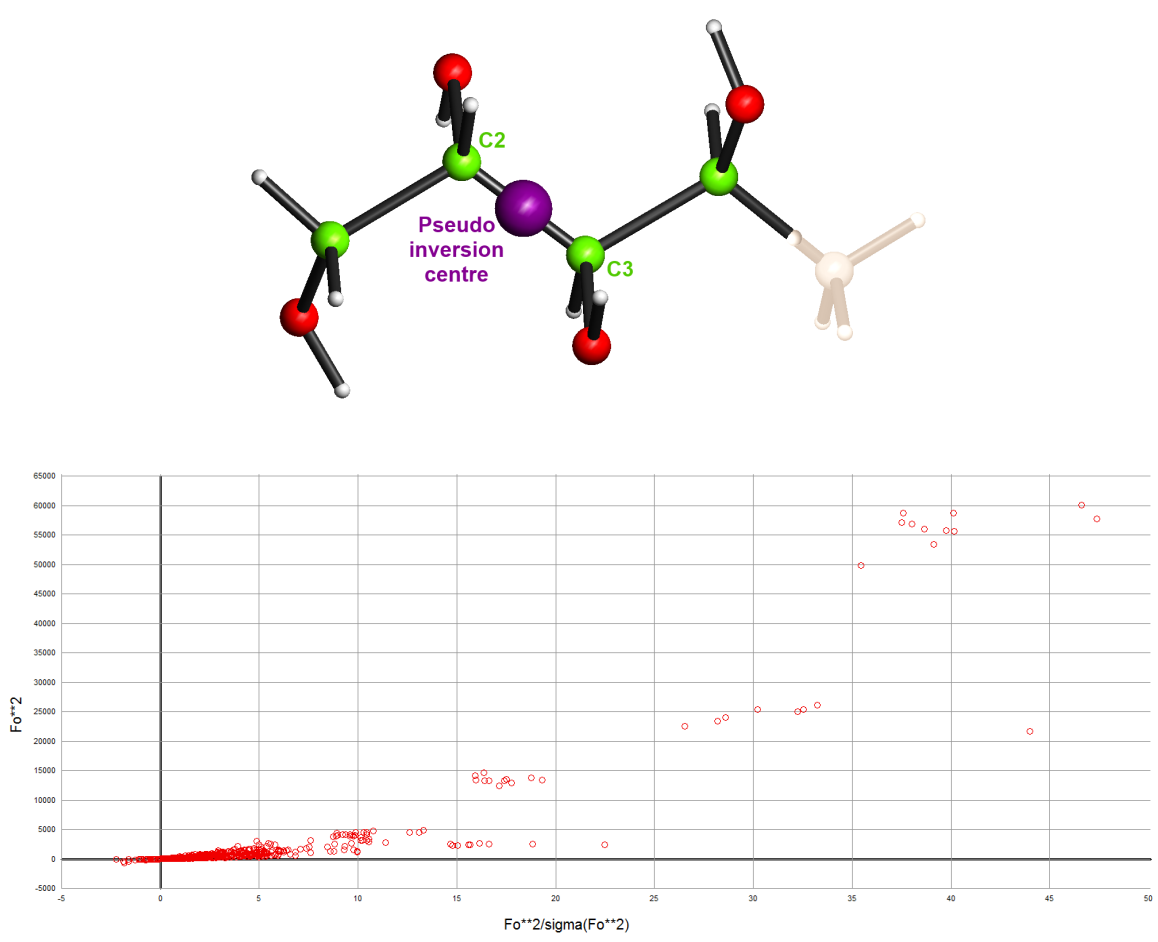

Figure 8: 1-Deoxy-L-arabinitol showing the inversion centre (top) with the intensity of the systematic absences shown for the full sphere data collected at $100 \mathrm{~K}$ (below). The fragment of the molecule which breaks the symmetry is shown on the right as a shadow together with the hydrogen atom generated by the inversion. All other atoms match well, however, and refinement of the structure in $\mathrm{I}_{1} /$ a modeling the symmetry breaking methyl group as 50\% occupied gives a good agreement with the data. Despite this, the systematic absences are clearly visible as they have a considerably larger magnitude than the most negative systematically absent reflections. 
it is clear that in the case of the high symmetry 1-deoxy-arabinitol collecting a full sphere of data is a poor use of resources. In this case, the hemisphere containing Friedel pairs would seem to give a perfectly adequate result so long as the reflections are significantly well determined. This study sought to come up with some general guidelines for non-expert users to replace the myriad of myths based on unsubstantiated heresay: here are the conclusions based on this study presented.

1. Although it is possible to determine the absolute structure without full Friedel coverage, these results can be misleading so a special effort should always be made to collect the Friedel pairs.

2. A small amount of additional redundancy can significantly improve the determination of the Flack " $x$ " parameter. From the study of 1-deoxyarabinitol, it would seem that collecting a redundancy of about five (based on the non-centrosymmetric point group) is a suitable compromise between wasting resources and improving results.

3. Redundancy is more important than $I / \sigma$, as long as the data collected are sufficiently strong. If the data are too weak, the results will be compromised. A mean $I / \sigma$ of $40-60$ over the whole dataset gave a good result, more than would perhaps have been expected.

4. It is favourable, barring issues with phase transitions etc., to collect data at the lowest temperature reasonably possible. There is very little additional expense involved in running a sample at $100 \mathrm{~K}$ rather than $200 \mathrm{~K}$, but there is a noticeable improvement in the determination Flack " $x$ " parameter.

5. When instrument time is available, collecting data for twice as long is only going to give a marginal improvement in the determination of the Flack " $x$ " parameter. It is generally better, therefore, to collect data on two different crystals instead of spending all the time on one. This has many advantages, including reducing the probability of getting a partial data collection due to instrumental factors. Perhaps most importantly though, it yields two essentially independent measurements of the same parameter which will also help to verify that the crystal is representative of the bulk. 


\section{Experimental}

\subsection{Data Collection - Tartaric Acid}

A $0.04 \times 0.17 \times 0.32 \mathrm{~mm}$ clear colourless crystal of D-tartaric acid was selected and placed in a droplet of perfluoro polyether oil. The crystal was then mounted on a $22 \mu \mathrm{m}$ MiTeGen MicroMount using the oil-drop technique before the crystal was placed directly into the dry nitrogen flow of an Oxford CryoSystems Cryostream 700 plus $^{26}$ at $150 \mathrm{~K}$ mounted on a (Rigaku) Oxford Diffraction SuperNova A diffractometer.

The CrysAlisPro Software was then used to build a strategy that was $100 \%$ complete to $0.8 \AA$ with copper radiation $(\lambda=1.54184 \AA)$ assuming the point group of $2 / \mathrm{m}$. Extra data were then added stepwise to increase the coverage first to 2 , then to 1 . Uncorrelated images were then collected $1 \mathrm{~s}$ for the low angle data and $4 \mathrm{~s}$ for the high angle data.

\subsection{Data Collection - 1-deoxy-L-arabinitol}

A $0.05 \times 0.06 \times 0.08 \mathrm{~mm}$ clear colourless crystal of 1-deoxy-L-arabinitol was selected and placed in a droplet of high viscosity perfluoro polyether oil. The crystal was then mounted on a $50 \mu \mathrm{m}$ MiTeGen MicroMount using the oil-drop technique. The crystal was then placed directly into the dry nitrogen flow of an Oxford CryoSystems Cryostream 700 plus ${ }^{26}$ at $300 \mathrm{~K}$ mounted on a (Rigaku) Oxford Diffraction SuperNova A diffractometer. The crystal was then cooled to $150 \mathrm{~K}$ at $300 \mathrm{~K} / \mathrm{hr}$ where data were collected.

The CrysAlisPro Software was then used to build a strategy that was $100 \%$ complete to $0.8 \AA$ with copper radiation $(\lambda=1.54184 \AA)$ assuming the point group of $4 / \mathrm{m}$. Extra data were then added stepwise to increase the coverage first to point group 4, then to 2 and finally to 1 . Uncorrelated images were then collected at $100 \mathrm{~K}$ with $1 \mathrm{~s}$ for the low angle data and $4 \mathrm{~s}$ for the high angle data. On completion similar datasets were collected at $200 \mathrm{~K}$ and then $150 \mathrm{~K}$ with 20 mins stabilisation time at each new temperature. Two further data collections were carried out at $150 \mathrm{~K}$ with extended acquisition times $(2 \mathrm{~s}$ and $8 \mathrm{~s}$ for the low angle and high angle data respectively then $4 \mathrm{~s}$ and $16 \mathrm{~s}$ for the low angle and high angle data).

The crystal was then warmed to $250 \mathrm{~K}$ at $300 \mathrm{~K} / \mathrm{hr}$ where another data collection was carried out using the same strategy ( $1 \mathrm{~s}$ and $4 \mathrm{~s}$ for the low angle and high angle data respectively). On completion, the crystal was cooled to $150 \mathrm{~K}$ at $300 \mathrm{~K} / \mathrm{hr}$ where another similar data collection was carried out, 
however, the generator power was reduced from $40 \mathrm{~W}$ to $20 \mathrm{~W}$ by reducing the current from $0.8 \mathrm{~mA}$ to $0.4 \mathrm{~mA}$.

On completion, the crystal was warmed to $300 \mathrm{~K}$ at $300 \mathrm{~K} / \mathrm{hr}$ where one final data collection was carried out as before at full power (with $1 \mathrm{~s}$ for the low angle and and $4 \mathrm{~s}$ for the high angle data).

\subsection{Data Processing and Structure Refinement}

The tartaric acid dataset was processed automatically using CrysAlisPro at the end of the collection. These data were then refinalised setting the Friedel pairs to non-equivalent and applying a $20 \% R_{\text {int }}$ frame filter to remove outliers. Data were merged and scaled assuming $\mathrm{P} 2_{1}$ symmetry. The data were then refinalised again applying run filters to select only the data of the desired point group $(2$ and $2 / m)$.

The data from the full sphere were then solved using SUPERFLIP ${ }^{27}$ and refined with full matrix least-squares against $F^{2}$ with anisotropic displacement ellipsoids using CRYstals. ${ }^{28,29}$ All hydrogen atoms were visible in the difference Fourier map, but were added at geometric positions where carbon was the parent atom. All hydrogen atoms were then refined with restraints prior to inclusion in the refinement as a riding model in the usual manner. ${ }^{30}$ The Flack $x$ parameter was also included in the refinement ${ }^{23}$ and the weighting scheme was optimised ${ }^{31}$ before refinement to convergence. This structure then provided a model which was refined against the other two datasets.

For the 1-deoxy-arabinitol data, the dataset at each temperature point was processed automatically using CrysAlisPro at the end of the collection. These were then refinalised setting the Friedel pairs to non-equivalent and applying a $20 \% R_{\text {int }}$ frame filter to remove outliers. Data were merged and scaled assuming $14{ }_{1}$ symmetry. Each dataset was then refinalised again applying run filters to select only the data of the desired point group $(4 / m, 4$ and 2).

The data from the $100 \mathrm{~K}$ full sphere experiment was then solved ab initio using ShelXS ${ }^{32}$ and refined with full matrix least-squares against on $F^{2}$ with anisotropic displacement ellipsoids using CRYSTALs. ${ }^{28,29}$ All hydrogen atoms were visible in the difference Fourier map, but were added at geometric positions where carbon was the parent atom. All hydrogen atoms were then refined with restraints prior to inclusion in the refinement as a riding model in the usual manner. ${ }^{30}$ The Flack $x$ parameter was also included in the refinement ${ }^{23}$ and the weighting scheme was optimised ${ }^{31}$ before refinement to convergence. 
This structure provided a model which was refined against each different dataset. First, each was refined with twelve cycles of least-squares optimising the scale factor, atomic positions, displacement ellipsoids and Flack $x$ parameter applying a $-3 \sigma$ intensity filter. Then the hydrogen atoms were optimised separately with restraints before they were added back into the refinement using the riding model and a further twelve least-squares cycles carried out. The reflection weights were optimised using the modified-Sheldrick weighting scheme (Type 16) then two further blocks of twelve cycles of least-squares separated by a second hydrogen refinement were carried out. In general, the Goodness of Fit was close to unity and the refinement was stable. However, for many of the $4 / m$ datasets, this was not the case, so the weighting scheme was re-optimised and the two blocks of twelve cycles of least-squares separated by a second hydrogen refinement were repeated. In each case, the Goodness of Fit and convergence were within acceptable tolerances.

\section{Acknowledgements}

The authors would like to thank James E. Thomson for the tartaric acid sample, Zoltan A. Gal and David J. Watkin for helpful discussions, and Users of the Oxford Chemical Crystallography Service for continued inspiration.

\section{References}

[1] L. Sohnke, Enlwicklung einer Theorie der Kristallstruktur, Leipzig, 1879 .

[2] L. Fábián, C. P. Brock, A list of organic kryptoracemates, Acta Crystallogr. B66 (2010) 94-103.

[3] G. Friedel, Sur les symétries cristallines que peut révéler la diffraction des rayons X, C.R. Acad. Sci. Paris 157 (1913) 1533-1536.

[4] J. M. Bijvoet, A. F. Peerdeman, A. J. van Bommel, Determination of the absolute configuration of optically active compounds by means of X-rays, Nat. 168 (1951) 271-273.

[5] W. C. Hamilton, Significance tests on the crystallographic R factor, Acta Crystallogr. 18 (1965) 502-510. 
[6] D. Rodgers, On the application of Hamilton's ratio test to the assignment of absolute configuration and an alternative test, Acta Crystallogr. A37 (1981) 734-741.

[7] H. D. Flack, On enantiomorph-polarity estimation, Acta Crystallogr. A39 (1983) 876-881.

[8] R. W. W. Hooft, L. H. Straver, A. L. Spek, Determination of absolute structure using Bayesian statistics on Bijvoet differences, J. Appl. Crystallogr. 41 (2008) 96-103.

[9] R. W. W. Hooft, L. H. Straver, A. L. Spek, Probability plots based on Student's t-distribution, Acta Crystallogr. A65 (2009) 319-321.

[10] R. W. W. Hooft, L. H. Straver, A. L. Spek, Using the t-distribution to improve the absolute structure assignment with likelihood calculations, J. Appl. Crystallogr. 43 (2010) 665-668.

[11] S. Parsons, P. Pattison, H. D. Flack, Analysing Friedel averages and differences, Acta Crystallogr. A68 (2012) 736-749.

[12] S. Parsons, H. D. Flack, T. Wagner, Use of intensity quotients and differences in absolute structure refinement, Acta Crystallogr. B69 (2013) 249-259.

[13] D. J. Watkin, R. I. Cooper, Why direct and post-refinement determinations of absolute structure may give different results, Acta Crystallogr. B72 (2016) 661-683.

[14] H. D. Flack, G. Bernardinelli, The use of X-ray crystallography to determine absolute configuration, Chirality 20 (2008) 681-690.

[15] L. Pasteur, Mémoire sur la relation qui peut exister entre la forme cristalline et la composition chimique, et sur la cause de la polarisation rotatoire, C. R. Acad. Sci. Paris 26 (1848) 535-538.

[16] L. Pasteur, Sur les relations qui peuvent exister entre la forme cristalline, la composition chimique et le sens de la polarisation rotatoire, Anal. Chim. Phys. 24 (1848) 442-459.

[17] A. L. Thompson, D. J. Watkin, X-ray crystallography and chirality: Understanding the limitations, Tet. Asymm. 20 (2009) 712-717. 
[18] G. Bernardinelli, H. D. Flack, Least-squares absolute-structure refinement. a case study of the effect of absorption correction, data region, stability constant and neglect of light atoms, Acta Crystallogr. A43 (1987) $75-78$.

[19] S. F. Jenkinson, F. P. Cruz, K. V. Booth, G. W. J. Fleet, K. Izumori, C.-Y. Yu, D. J. Watkin, 1-Deoxy-D-arabinitol, Acta Crystallogr. 64 (2008) o1010-o1011.

[20] A. E. Goeta, J. A. K. Howard, Low temperature single crystal X-ray diffraction: advantages, instrumentation and applications, Chem. Soc. Rev. 33 (2004) 490-500.

[21] H. D. Flack, U. Shmueli, Mean-square Friedel difference in P1, Acta Crystallogr. A63 (2007) 257-265.

[22] H. D. Flack, G. Bernardinelli, Applications and properties of the Bijvoet intensity ratio, Acta Crystallogr. A64 (2008) 484-493.

[23] A. L. Thompson, D. J. Watkin, CRYstals enhancements: absolute structure determination, J. Appl. Crystallogr. 44 (2011) 1017-1022.

[24] H. D. Flack, G. Bernardinelli, D. A. Clemente, A. Linden, A. L. Spek, Centrosymmetric and pseudo-centrosymmetric structures refined as non-centrosymmetric, Acta Crystallogr. B62 (2006) 695-701.

[25] D. W. J. Cruickshank, W. S. McDonald, Parameter errors in polar space groups caused by neglect of anomalous scattering, Acta Crystallogr. 23 (1967) 9-11.

[26] J. Cosier, A. M. Glazer, A nitrogen-gas-stream cryostat for general X-ray diffraction studies, J. Appl. Crystallogr. 19 (1986) 105-107.

[27] L. Palatinus, G. Chapuis, SuperFliP a computer program for the solution of crystal structures by charge flipping in arbitrary dimensions, J. Appl. Crystallogr. 40 (2007) 786-790.

[28] P. W. Betteridge, J. R. Carruthers, R. I. Cooper, K. Prout, D. J. Watkin, CRYstals version 12: software for guided crystal structure analysis, J. Appl. Crystallogr. 36, (2003) 1487. 
[29] P. Parois, R. I. Cooper, A. L. Thompson, Crystal structures of increasingly large molecules: meeting the challenges with CRYSTALS software, Chem. Cent. J. 9 (2015) 30.

[30] R. I. Cooper, A. L. Thompson, D. J. Watkin, CRYstals enhancements: dealing with hydrogen atoms in refinement, J. Appl. Crystallogr. 43 (2010) 1100-1107.

[31] E. Prince, Mathematical Techniques in Crystallography and Materials Science, Springer-Verlag, New York, 1982.

[32] G. M. Sheldrick, A short history of ShelX, Acta Crystallogr. A64 (2008) 112-122. 